\title{
Tracing the Sgr Stream with 2MASS
}

\section{Detection of Stream stars around Outer Halo globular clusters ${ }^{\star}$}

\author{
M. Bellazzini ${ }^{1}$, R. Ibata ${ }^{2}$, F. R. Ferraro ${ }^{3}$, and V. Testa ${ }^{4}$ \\ 1 INAF - Osservatorio Astronomico di Bologna, via Ranzani 1, 40127 Bologna, Italy \\ 2 Observatoire de Strasbourg, 67000 Strasbourg, France \\ e-mail: ibata@astro.u-strasbg.fr \\ 3 Dipartimento di Astronomia, Università di Bologna, via Ranzani 1, 40127 Bologna, Italy \\ e-mail: ferraro@bo.astro.it \\ ${ }^{4}$ INAF - Osservatorio Astronomico di Roma, via Frascati 33, 00040 Monte Porzio Catone, Italy \\ e-mail: testa@mporzio.astro.it
}

Received 24 February 2003 / Accepted 24 April 2003

\begin{abstract}
We use infrared Color Magnitude Diagrams from the 2-Micron All-Sky Survey (2MASS) to search for stars belonging to the tidal stream of the Sagittarius dwarf spheroidal galaxy (Sgr dSph) around selected Galactic globular clusters. Statistically significant detections are presented for the cases of Pal 12 and NGC 4147, strongly supporting the idea that these clusters are associated with the Sgr Stream and that they were previous members of the Sgr dSph galaxy.
\end{abstract}

Key words. Galaxy: halo - Galaxy: structure - globular clusters: individual: Pal 12, NGC 4147

\section{Introduction}

There is clear evidence that the Sagittarius dwarf Spheroidal galaxy (Sgr dSph; Ibata et al. 1994, 1997) has lost (and is currently loosing) a significant fraction of its stars under the strain of the Milky Way tidal field. The lost stars seem able to remain coherently aligned with the orbital path of the Sgr dSph as a long-lived (>few Gyr; see Johnston et al. 1999; Ibata \& Lewis 1998) stellar relic, known as the Sgr Stream. Parts of the Sgr Stream have been detected by many different groups and with many different techniques, up to $>100^{\circ}$ away from the main body of the galaxy (Fahlman et al. 1996; Mateo et al. 1998; Majewski et al. 1999; Helmi et al. 1999; Ibata et al. 2001a, 2002; Kundu et al. 2002; Martínez-Delgado et al. 2001; Vivas et al. 2001; Yanny et al. 2000; Ivezic et al. 2000). Direct identifications, based on the similarity of the CMD in the main body of the galaxy and in portions of the Stream $\sim 160^{\circ}$ away in galactic longitude have been provided by Newberg et al. (2002).

Send offprint requests to: M. Bellazzini, e-mail: bellazzini@bo.astro.it

* This publication makes use of data products from the Two Micron All Sky Survey, which is a joint project of the University of Massachusetts and the Infrared Processing and Analysis Center/California Institute of Technology, funded by the National Aeronautics and Space Administration and the National Science Foundation.
Ibata \& Lewis (1998, hereafter IL98) and Ibata et al. (2001a) have simulated the evolution of the Sgr dSph over several orbital periods $(P \sim 1 \mathrm{Gyr})$, computing the orbit of the galaxy as well as the phase-space distribution of the debris under different assumptions about the flattening of the CDM halo. The initial conditions of the simulations were based on the known position and radial velocity of $\mathrm{Sgr} \mathrm{dSph}$ and on its proper motion as estimated by Ibata et al. (1997, 2001a). The orbit has a planar rosette structure, with the pole of the orbit located at $\left[\ell=90^{\circ}, b=-13^{\circ}\right]$ (i.e. a nearly polar orbit), and peri- and apo-Galactic distances of $15 \mathrm{kpc}$ and $60 \mathrm{kpc}$ respectively. The derived orbit has successfully been used to predict the observed position (Ibata et al. 2001a,b) and radial velocity (Yanny et al. 2003) of the Sgr Stream.

In a previous paper (Bellazzini et al. 2003, hereafter BFI03) we compared the IL98 orbit during the last orbital period with a subset of galactic globular clusters, namely those having $10 \mathrm{kpc} \leq R_{\mathrm{GC}} \leq 40 \mathrm{kpc}$ which we termed Outer Halo $(\mathrm{OH})$ globulars, in the phase-space of Galactocentric cartesian coordinates and radial velocity $\left(X, Y, Z, V_{\mathrm{r}}\right.$ : see BFI03 for further details). We found that the region of phase-space occupied by the Sgr Stream hosts a significant overdensity of OH clusters, thus some of them have to be physically associated with the Stream and were former members of the Sgr dSph. While the association of at least $4 \mathrm{OH}$ clusters with the Stream is quite firmly established, the result is statistical in nature and we cannot confirm the actual membership of individual clusters with 
the adopted technique. Hence, we simply provided a list of candidate Stream members ranked according to their proximity to the Sgr orbit in the considered phase-space. Strong support for individual associations may be provided by a direct detection of Sgr Stream stars around a candidate cluster and located at the same distance from us, as provided, for instance, by Martínez-Delgado et al. (2002) in the case of our first-ranked candidate Pal 12 (see also Dinescu et al. 2000).

This kind of search requires the sampling of wide areas of sky, since the Stream is a very low surface brightness structure, and the use of suitable tracer stars that can be singled out from the strong contamination provided by ordinary Galactic field stars (see Ibata et al. 2001a; Yanny et al. 2000; Ivezic et al. 2000; Newberg et al. 2002; Vivas et al. 2001; Ibata et al. 2002 for the different tracers used to date). Here we use stars in the upper Red Giant Branch (RGB) from the near infrared Color-Magnitude Diagrams (CMD) of wide areas of sky provided by the 2-Micron All-Sky Survey (2MASS) to search for Stream stars around the top-ranked candidates listed in Table 1 of BFI03. Sgr Stream stars at the "right" distance are detected around Pal 12, thus confirming the result by Martínez-Delgado et al. (2002), and around NGC 4147, a completely new detection. The present study has to be considered as a preliminary "pilot" analysis. We will perform a deeper and more complete search once the final all-sky 2MASS catalogue is released.

The plan of the paper is the following: in Sect. 2 we present the data and the method; in Sects. 3 and 4 we describe the results obtained for Pal 12 and NGC 4147, respectively; in Sect. 5 we deal briefly with the case of the other considered candidates; Sect. 6 gives a short summary and a discussion of the reported results.

\section{Data and method}

All the photometric data are from the Point Source Catalogue (PSC) of the Second Incremental Data Release of $2 \mathrm{MASS}^{1}$ and consist of calibrated $J, H, K_{\mathrm{S}}{ }^{2}$ magnitudes and positions of sources classified as stellar and without any detected contamination from other nearby sources. Magnitudes and colors of all the retrieved sources have been corrected for interstellar extinction by interpolating in the IRAS-DIRBE reddening maps of Schlegel et al. (1998) and adopting the extinction laws by Savage \& Mathis (1979). The present sky coverage of 2MASS is quite patchy, hence there are regions of sky that cannot be scrutinized. We typically select areas of $20^{\circ} \times 20^{\circ}$ toward interesting directions, but in all cases large unobserved zones are included in the selected areas. Typically, the effective sampled area ranges from $\sim \frac{1}{4}$ to $\sim \frac{2}{3}$ of the selected area (see, for example, Figs. 4 and 5, below). Distances and other parameters for globular clusters are taken from the compilation by Harris (1996).

Figure 1 displays the $K, J-K \mathrm{CMD}$ of a $4^{\circ} \times 4^{\circ}$ field centered on the point of maximum density of the main body

\footnotetext{
${ }^{1}$ See Cutri et al. (2000), Explanatory Supplement to the 2MASS Second Incremental Data Release,

http://www.ipac. caltech.edu/2mass/releases/second/ doc/expsup.html

${ }^{2}$ From now on we drop the $S$ index from $K_{\mathrm{S}}$, for brevity.
}

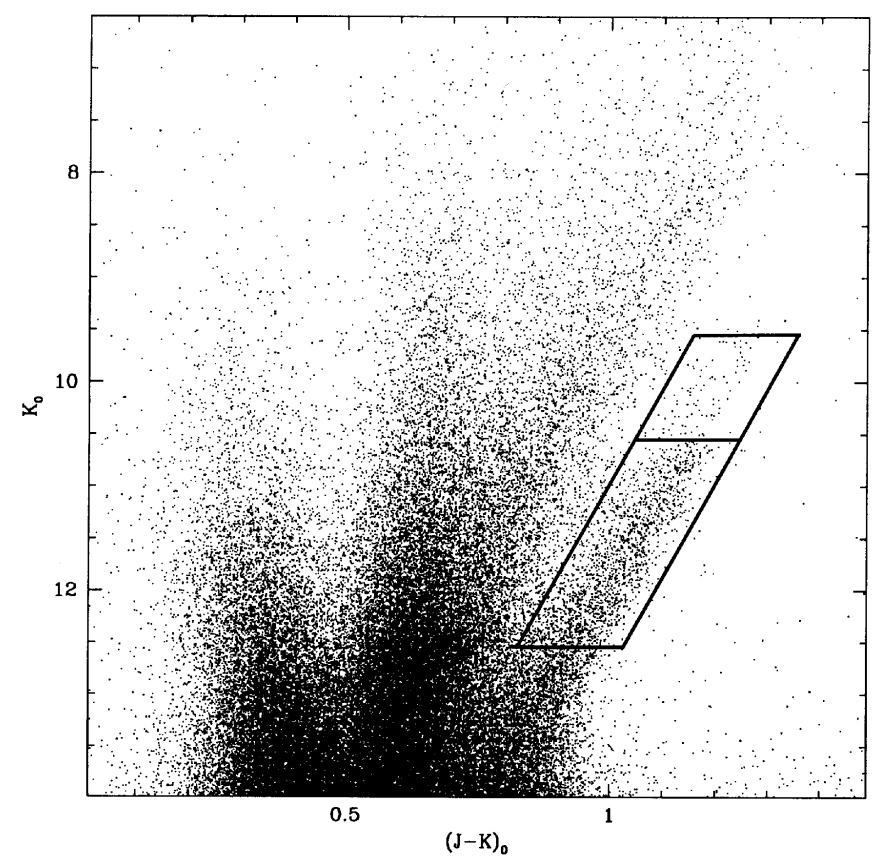

Fig. 1. $K_{\mathrm{S}}, J-K_{\mathrm{S}} \mathrm{CMD}$ of a $\sim 4^{\circ} \times 4^{\circ}$ area around the center of the Sgr dSph galaxy. The upper RGB of Sgr stands out very clearly at $J-K_{\mathrm{S}}>1$, to the red of all the features formed by galactic field stars. The lower box encloses the Sgr RGB stars, the upper box encloses Sgr bright AGB stars.

of the Sgr dSph. A similar field has been used by Cole (2001) to study the metallicity of the galaxy and virtually the same field has been considered by Alard (2001) in his analysis of the metallicity gradient. As noted by these authors the brighter part of the RGB of the Sgr dSph stands out very clearly in this diagram, to the red of any feature due to galactic field stars in the region $(J-K)_{0}>0.8$ and $K_{0} \lesssim 12$ (see Cole 2001 and Alard 2001 for discussion of the field features in the CMD). The lower part of the reported box encloses the more clearly visible part of the Sgr RGB. The upper (smaller) sector of the box encloses bright Asymptotic Giant Branch (AGB) stars of Sgr. We will use only RGB stars as tracers of the Sgr stellar population but we maintain also the upper sector of the box to check also if rarer AGB stars fall in our selection box. The reddest Sgr stars from 2MASS have been already used by Ibata et al. (2002) for a pole-count analysis that detected an overdensity of such stars in the plane of the sky in the directions predicted by the IL98 orbit.

The detection method is quite simple: since Sgr RGB stars stand out clearly against galactic field stars in the considered CMD, we will look if similar stars can be found also in wide fields surrounding $\mathrm{OH}$ globulars that are candidate stream members according to BFI03. The RGB feature, if present, has to be shifted in magnitude by the difference in true distance moduli between the Sgr dSph and the considered cluster $\left(\Delta \mu_{0}\right)$. Therefore, we will simply report the Selection Box (SB) shown in Fig. 1 in the CMDs of the selected area and of a suitable Control Field (CF) applying a shift by $\Delta \mu_{0}$. Then we will check if there is a significant overdensity of stars in the SB of the selected area with respect to the CF. It is important to note that 
the SB has been constructed to include also the bluest stars in the Sgr RGB, in order to remain sensitive to the searched feature notwithstanding the effect of the metallicity/population radial gradient observed in Sgr (Alard 2001).

Since we are looking for very low-surface-brightness structures, it is of the utmost importance to avoid any possible source of contamination. To this end, within the SB we will select only stars having $(J-K)_{0}>0.95$, to avoid the inclusion of the reddest stars from the local $\mathrm{M}$ dwarf plume at $(J-K)_{0} \sim 0.8$, and we will reject stars with $K_{0}>12$, since in this range of magnitude a sparse cloud of red sources (possibly misclassified galaxies ${ }^{3}$ ) provides additional contamination. For this reason, the adopted technique is nearly insensitive to the feature of interest for $\Delta \mu_{0} \gtrsim+0.5$, i.e. the Sgr Stream RGB can be detected only up to a distance of $\sim 32 \mathrm{kpc}$ from us. In some cases (e.g., NGC 4147) the considered clusters fall in an unobserved zone of the selected area, thus contamination of the SB by cluster stars cannot occur. In all other considered cases we checked the CMD of the stars in the immediate vicinity of the cluster (up to $1^{\circ}$ from its center) and we never found the case of a possible cluster star falling in the SB. This is due to the fact that the considered clusters are significantly more metal poor than the main population of Sgr (see Cole 2001; Monaco et al. 2002 and references therein), hence their RGBs are much bluer and fall within the Galactic field features of the CMD.

In this first attempt we considered the first six clusters of the ranked list of candidates of BFI03, these are, in order of increasing phase-space distance from the IL98 orbit: Pal 12, NGC 4147, Pal 5, NGC 5634, NGC 5053 and Ter 3. Searching the PSC catalogue we have found suitable selected areas for Pal 12 and NGC 4147. The present sky coverage of 2MASS around NGC 5634, Pal 5 and NGC 5053 is insufficient to obtain an effective sampled area useful for the present purpose. Finally, we recently realized (Marsakov, private communication) that the 2003 version of the Harris (1996) catalogue reports significantly revised estimates of the reddening and distance modulus of Ter $3\left[E(B-V)=0.72 ;(m-M)_{V}=16.61\right]$ with respect to the 1996 version that was used by BFI03 $\left[E(B-V)=0.32 ;(m-M)_{V}=18.00\right]$. The new distance estimate places the cluster quite far from the Sgr orbit. As a consequence, Ter 3 was removed from the list of candidates and it is not considered in the present analysis. Thus, the test remains to be done for Pal 5, NGC 5634 and NGC 5053 (these cases are shortly discussed in Sect. 5, below), while in the following two sections we show the results we have obtained for the cases of Pal 12 and NGC 4147.

\section{Pal 12}

To search for Sgr Stream stars around this cluster, whose galactic coordinates are $(l ; b)=\left(30.512^{\circ} ;-47.681^{\circ}\right)$, we selected the area comprised in the ranges $20^{\circ} \leq l \leq 40^{\circ}$ and $-57^{\circ} \leq$ $b \leq-37^{\circ}$. The effectively sampled field is $71 \%$ of the selected area. In the upper panels of Fig. 2 the CMDs of the Pal 12 field is compared to the CMD of a suitable nearby Control Field

\footnotetext{
3 The feature is more evident in less crowded fields with respect to that shown in Fig. 1; see Figs. 2 and 3.
}

at similar galactic latitude $\left(80^{\circ} \leq l \leq 100^{\circ}\right.$ and $-55^{\circ} \leq b \leq$ $\left.-35^{\circ}\right)$. The difference in true distance modulus between Pal 12 and the core of the Sgr galaxy is $\Delta \mu_{0}=\mu_{0}(\mathrm{P} 12)-\mu_{0}(\mathrm{M} 54)=$ $-0.76 \pm 0.15$. Hence the SB shown in Fig. 2 has been shifted by $\Delta \mu_{0}=-0.76$ in $\mathrm{K}$ to search for the RGB feature at the distance of Pal 12.

Even from a visual inspection it is clear that Sgr RGB stars are present in the field surrounding the cluster and are missing in the CF. To quantify the observed difference we count the stars falling into the SB (with the additional conditions $(J-K)_{0}>0.95$ and $\left.K_{0} \leq 12.0\right)$ in the two CMDs $\left(N_{b}^{\text {clus }}\right.$ and $N_{b}^{\mathrm{CF}}$, for the cluster field and for the $\mathrm{CF}$, respectively). To take simultaneously into account the differences in sampled area and any possible difference due to galactic population gradients between the cluster fields and the CFs we normalize to the number of stars spanning the same magnitude range of the plotted SB and with colors $0.55<(J-K)_{0}<0.90$ $\left(N_{\text {norm }}^{\text {clus }}\right.$ and $\left.N_{\text {norm }}^{\mathrm{CF}}\right)$. Note, however, that all the results are fully confirmed if we normalize by the ratio of the sampled areas instead. In particular, the normalization ratios by number and by area are virtually identical in all of the considered cases. Defined $R=N_{\text {norm }}^{\text {clus }} / N_{\text {norm }}^{\mathrm{CF}}$, we compute the normalized difference:

$$
\Delta N_{\star}=N_{b}^{\text {clus }}-R \times N_{b}^{\mathrm{CF}}
$$

and its standard deviation, assuming Poisson errors in star counts:

$$
\sigma\left(\Delta N_{\star}\right)=\sqrt{N_{b}^{\text {clus }}+R^{2} \times N_{b}^{\mathrm{CF}}}
$$

In the present case $\Delta N_{\star}=53.5 \pm 10.9$ is obtained, that is, a very significant difference $(4.9 \sigma)$. The searched RGB feature is indeed present around $\mathrm{Pal} 12$, it is statistically significant and it is found at the right magnitude level.

To explore better the nature of the detected signal we repeat the counts shifting the SB in the range $\Delta \mu_{0}=-0.76 \pm 1.0$ at 0.04 mag steps. We plot the resulting $\Delta N_{\star}$ as a function of $\Delta \mu_{0}$ in the lower panel of Fig. 2. The normalized difference reaches a wide maximum in the range $-1.1 \leq \Delta \mu_{0} \leq-0.5$ and the characteristic $\Delta \mu_{0}$ of Pal 12 (indicated by a filled triangle in the top of the panel) falls right in the middle of the detected plateau. The plot has to be considered just a consistency test since the constraining power on distance of a sparsely populated upper RGB sequence is obviously not strong. Moreover, the signal remains significant over a wide range of $\Delta N_{\star}$ because, after all, the Sgr stars are present in the Pal 12 field and absent in the CF in this area of the CMD. Thus any position of the SB that includes some of the Sgr RGB stars provides a sensible difference with respect to the CF. The effects of this syndrome may be particularly strong in shifting the SB toward brighter magnitudes $\left(K_{0} \lesssim 10.5\right)$ since in this region the $\mathrm{CF}$ is virtually devoid of stars.

Notwithstanding the above caveats the detection of Sgr Stream stars around Pal 12 is clear and significant, confirming the findings by Martínez-Delgado et al. (2002). Given it's position in the phase-space with respect to the orbit of the Sgr dSph (see BFI03) and its physical location within the Sgr Stream, we conclude that Pal 12 presently belongs to the 

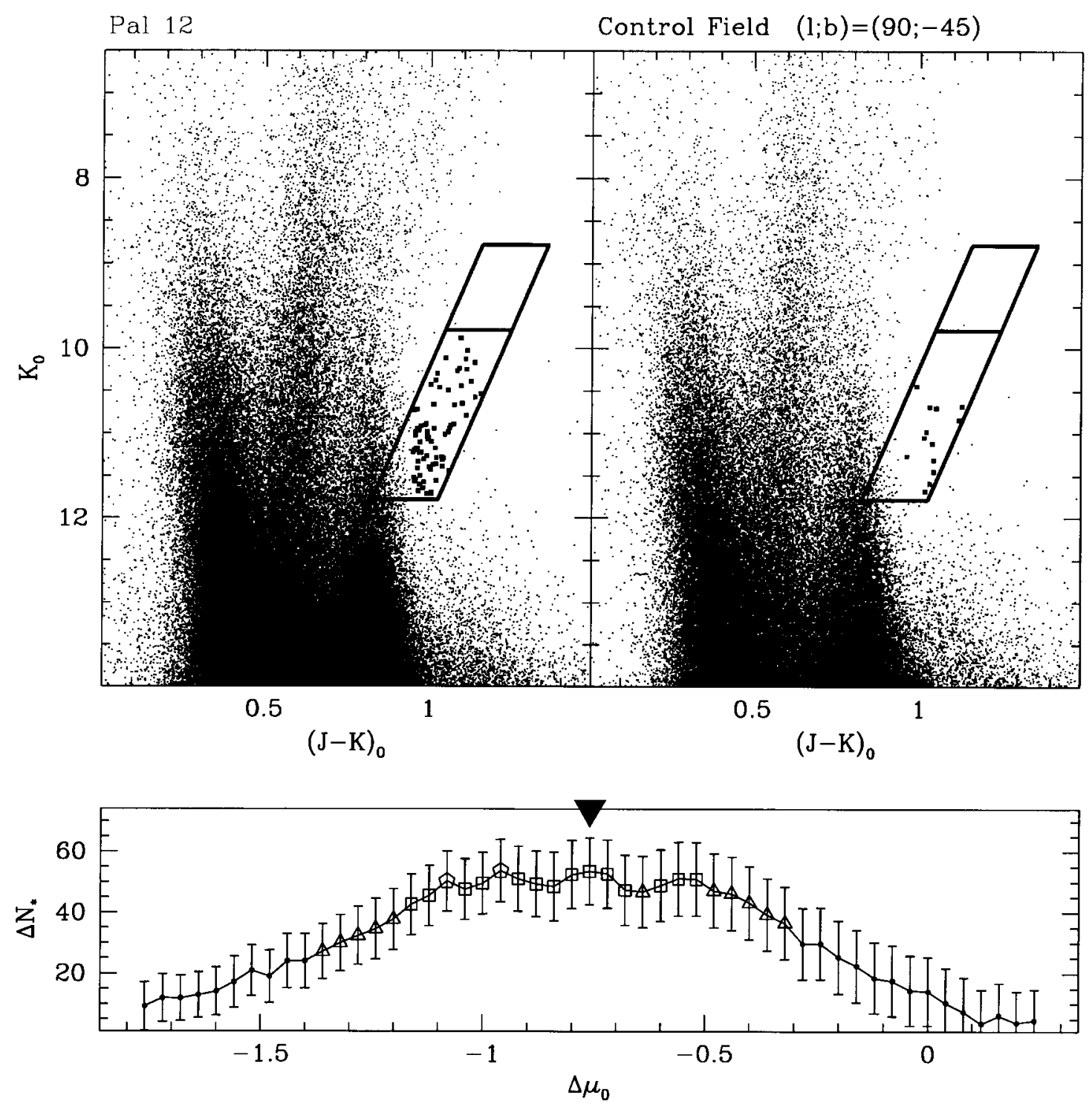

Fig. 2. Upper panels: the CMD of the selected area around the position of Pal 12 (left upper panel) and of a Control Field located at similar Galactic latitude. The reported Selection Box has been shifted by $\Delta \mu_{0}=-0.76$ with respect to Fig. 1, to displace it to the distance of Pal 12 . The selected stars are marked with heavier points. Lower panel: the normalized difference in the number of stars falling in the Selection Box as a function of $\Delta \mu_{0}$. Different symbols correspond to different values of $\Delta N_{\star}$ in units of $\sigma$ (filled points: $\Delta N_{\star} / \sigma<3$, triangles: $3 \leq N_{\star} / \sigma<4$, squares: $4 \leq N_{\star} / \sigma<5$, etc.). The large filled triangle on the top of the panel marks the assumed $\Delta \mu_{0}$ for Pal 12 .

Sgr Stream and that it was a former member of the globular cluster system of the Sgr dSph.

Even an educated guess of the surface brightness $(\Sigma)$ of the Sgr Stream at the considered locations would be of great interest for future modeling of the dynamical history of the dwarf galaxy. We obtain such an estimate by counting the number of stars in the SB having luminosities within 1 mag of the assumed RGB Tip ( $N_{\text {Tip }}^{\text {clus }}$ ), dividing by the sampled area and rescaling by the central surface brightness of Sgr dSph to the ratio of surface density of RGB Tip stars in the considered area and in the center of Sgr. The underlying assumption is that the stellar population in the center of the Sgr dSph and in the Sgr Stream are similar. The adopted method is widely used (see, for example, Kleyna et al. 1998; Mateo et al. 1991) and its physical basis is anchored on the Evolutionary Flux
Theorem (Renzini \& Buzzoni 1986; Renzini \& Fusi Pecci 1988; Renzini 1998). In the central $0.5 \times 0.5 \mathrm{deg}^{2}$ of Sgr dSph we find $N_{\text {Tip }}^{\text {clus }}=50$ stars within 1 mag from the RGB Tip and we assume that this corresponds to the central surface brightness of $\Sigma_{V}(0) \sim 25.4 \pm 0.3 \mathrm{mag} / \mathrm{arcsec}^{2}$ reported by Mateo (1998). The possible contamination by field stars is tentatively removed with the same technique used above, i.e. considering $N_{\star}^{\text {Tip }}=N_{\text {Tip }}^{\text {clus }}-R \times N_{\text {Tip }}^{\text {CF }}$ instead of $N_{\text {Tip }}^{\text {clus }}$ itself. Finally, we obtain for the average surface brightness in the Sgr Stream around Pal 12, $\Sigma_{V}=33.9 \pm 0.5 \mathrm{mag} / \mathrm{arcsec}^{2}$. The uncertainty has been estimated by 1000 Montecarlo simulations in which $\Sigma_{V}^{\mathrm{Sgr}}(0)$, $N_{\text {Tip }}^{\text {clus }}$ and $N_{\text {Tip }}^{\text {Sgr }}$ were simultaneously extracted from gaussian distributions having the observed values as mean and the reported $1-\sigma$ uncertainty as $\sigma$. 

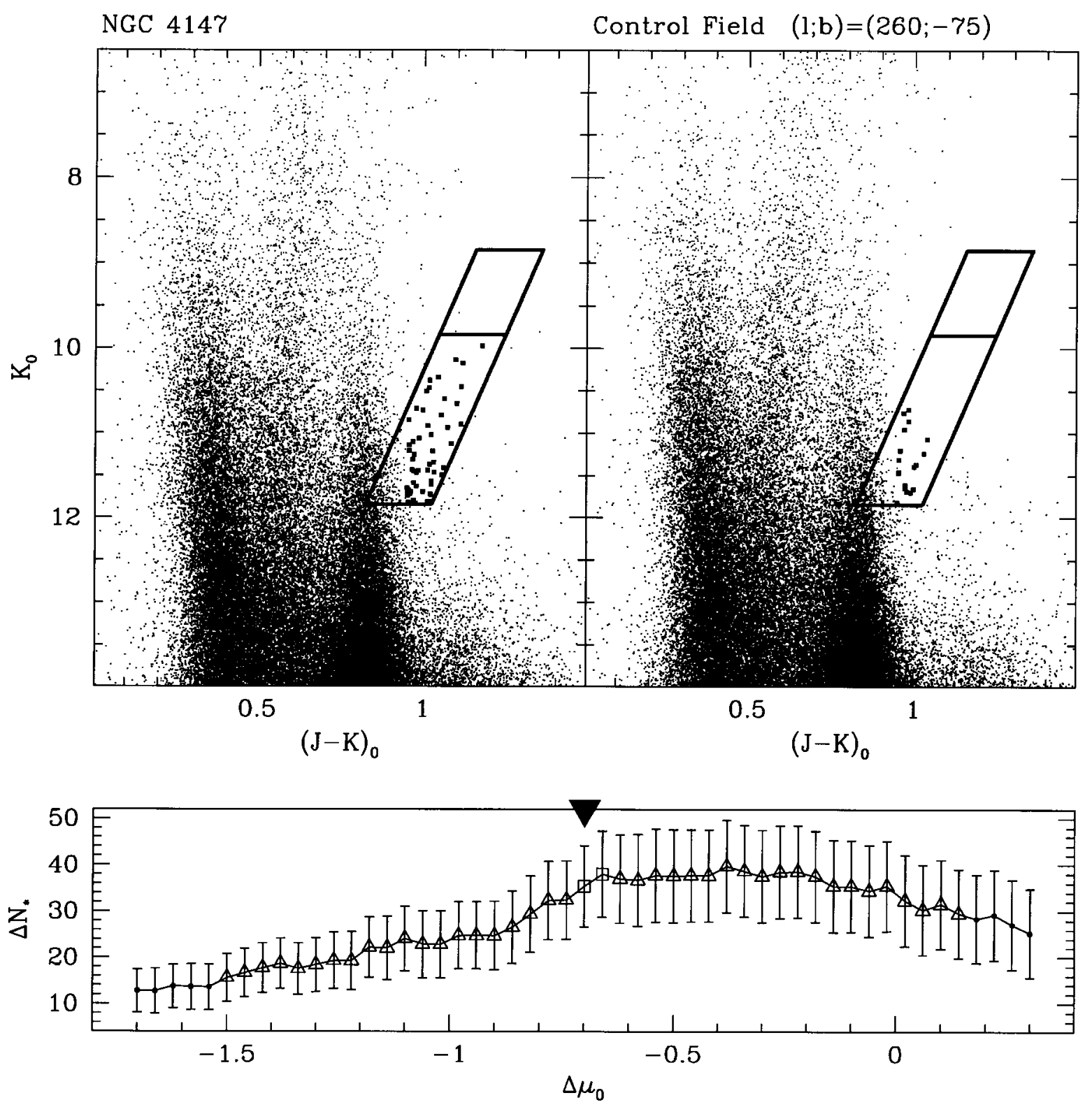

Fig. 3. The same as Fig. 2 for the selected area around the position of NGC 4147 and of a Control Field symmetrical with respect to the Galactic equator. $\Delta \mu_{0}=-0.70$, in this case.

\section{NGC 4147}

NGC $4147\left[(l ; b)=\left(252.850^{\circ} ; 77.189^{\circ}\right)\right]$ lies in a region of the sky $\left(220^{\circ} \lesssim l \lesssim 320^{\circ}\right)$ where the orbit of the Sgr dSph runs approximately at constant galactic latitude $\left(b \sim 75^{\circ}\right)$. We take advantage from this occurrence by taking a wide selected area comprised within $230^{\circ} \leq l \leq 290^{\circ}$ and $65^{\circ} \leq b \leq 85^{\circ}$. The effectively sampled field is $50 \%$ of the selected area. As Control Field we choose the region of the sky symmetric with respect to the galactic equator, i.e. $230^{\circ} \leq l \leq 290^{\circ}$ and $-85^{\circ} \leq b \leq-65^{\circ}$. The resulting CMDs are shown in Fig. 3, which is arranged in the same way and with the same symbols as Fig. 2, above. The difference in true distance modulus between NGC 4147 and the core of the Sgr galaxy is $\Delta \mu_{0}=\mu_{0}(N 4147)-\mu_{0}(M 54)=-0.70 \pm 0.15$ and the SB has been shifted accordingly in these panels with respect to Fig. 1.
Also in this case the Sgr RGB feature is quite evident and significant. The normalized difference is $\Delta N_{\star}=35.4 \pm 8.7$, a $4.0 \sigma$ signal. The lower panel of Fig. 3 shows a less pronounced maximum with respect to the Pal 12 case, probably due to the higher degree of contamination of the considered CMDs in the region $(J-K)_{0}>0.95$. The adopted $\Delta \mu_{0}$ for NGC 4147 falls at the "bright" edge of the plateau. However if we consider the normalized difference in terms of standard deviations $\left(N_{\star} / \sigma\right)$, the maximum signal is detected at $\Delta \mu_{0}=-0.70 \pm 0.04$, i.e. at the distance of NGC 4147.

Hence, it has to be concluded that also this cluster is immersed in the Sgr Stream and that it is physically associated with this structure.

Using the technique illustrated above we obtained $\Sigma_{V}=$ $34.0 \pm 0.6 \mathrm{mag} / \operatorname{arcsec}^{2}$ for the Sgr Stream surrounding 


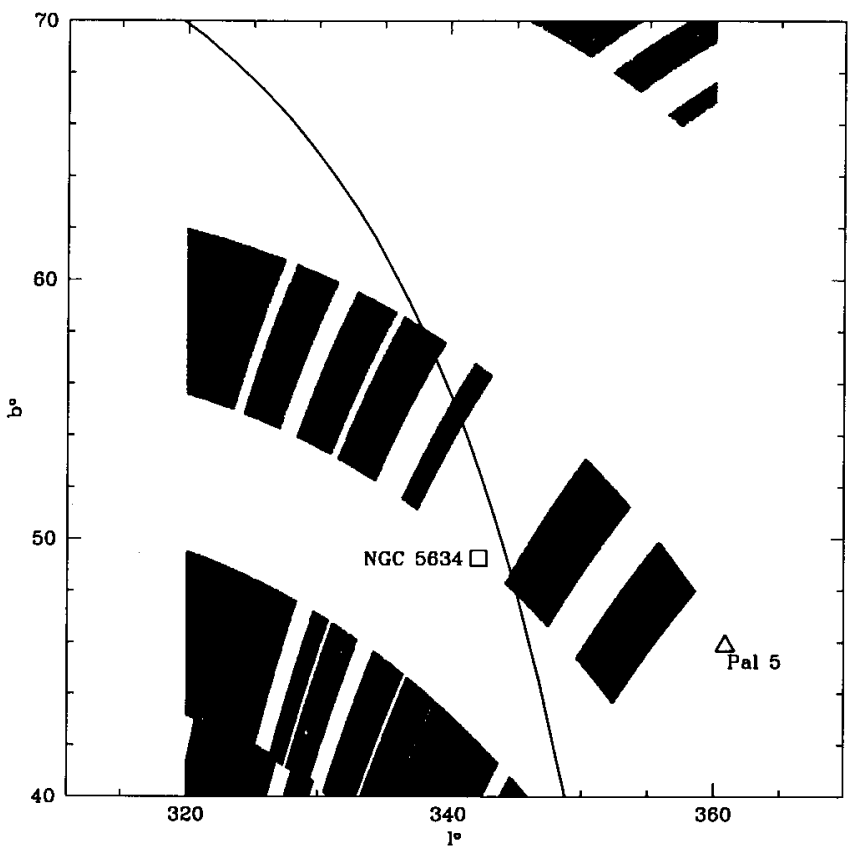

Fig. 4. The fields sampled by 2 MASS in the selected area around NGC 5634 and Pal 5 (in grey). The continuous line is the projection in the sky of the Sgr orbit by IL98.

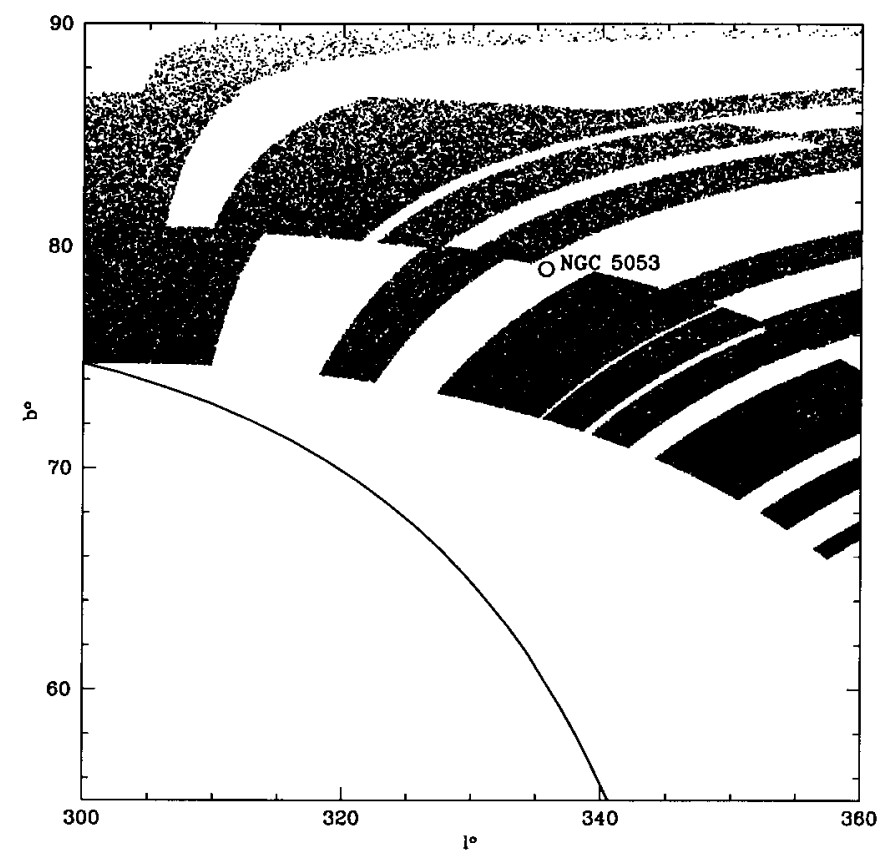

Fig. 5. The fields sampled by $2 \mathrm{MASS}$ in the selected area around NGC 5053 (in grey). The continuous line is the projection in the sky of the Sgr orbit by IL98.

NGC 4147. The comparison with the analogous estimate obtained in the region around Pal 12 suggests that the Stream maintains nearly constant surface brightness along its whole extension (see also Mateo et al. 1998).

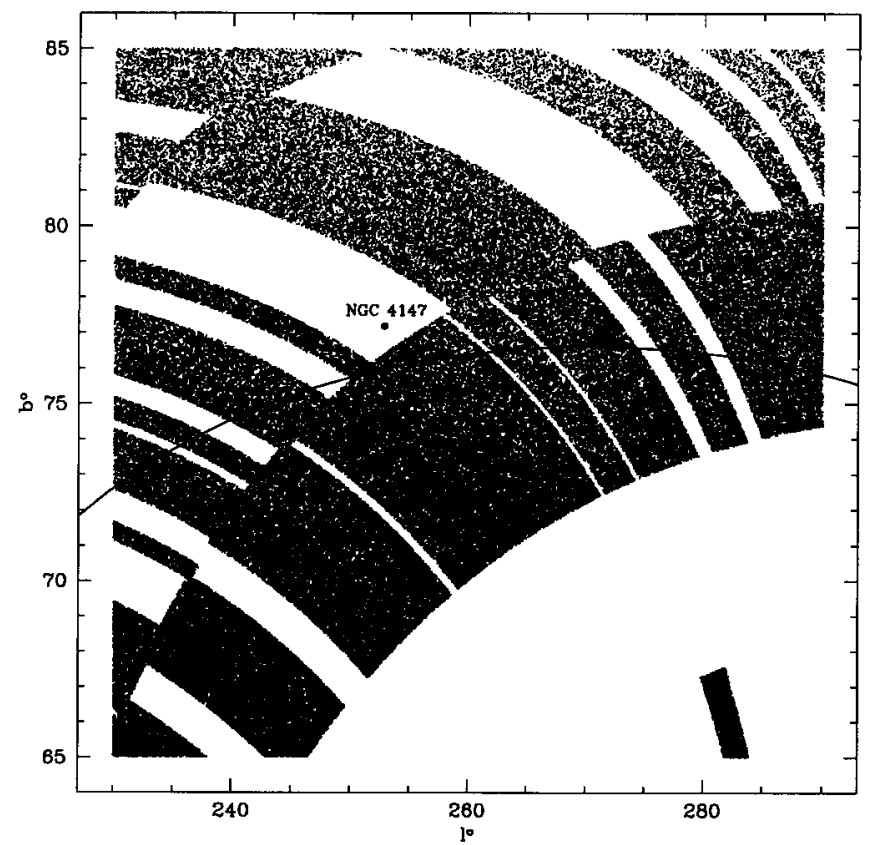

Fig. 6. The fields sampled by 2 MASS in the selected area around NGC 4147 (in grey). The continuous line is the projection in the sky of the Sgr orbit by IL98.

\section{Other cases}

In this section we shortly comment on the other candidates by BFI03 considered by the present study. In all cases we cannot reach a firm conclusion, either because of a too small effectively sampled area or because of problems with interstellar extinction.

NGC 5634 and Pal 5 are relatively nearby in the sky and have similar $\Delta \mu_{0}$, and thus may be studied simultaneously. Figure 4 shows that the coverage of 2MASS in this region of sky is quite poor and unfavorably placed with respect to the considered clusters and to the Sgr orbit. Hence, the absence of a significant detection in the selected area shown in Fig. 4 cannot be considered as evidence that the clusters are not associated with the Sgr Stream. Hopefully, the question should be resolved when future releases of the 2MASS catalogue are available.

The same problem plagues the application of the test to NGC 5053. In this case only a barely significant detection is obtained but the effective sampling is clearly insufficient and unfavorable (see Fig. 5).

In Fig. 6 we report, for comparison, the distribution of the sampled fields in the selected area around NGC 4147.

\section{Summary and conclusions}

We have examined the 2MASS $K_{0} ;(J-K)_{0}$ CMDs of wide areas of the sky around six Outer Halo globular clusters to search for stars belonging to the Sgr Stream and that are located approximately at the same distance as the considered clusters. The considered clusters were selected for their phase-space proximity to the orbit of the Sgr galaxy, according to BFI03. The dataset was found to be suitable for the application of the 
test to the cases of Pal 12 and NGC 4147. In both cases, an overdensity of tracer stars of similar heliocentric distance as the clusters, was detected at the $>4.5 \sigma$ level of significance with respect to Control Fields. Hence we have shown that both clusters are physically immersed in the Sgr Stream and are very likely members of this structure as well as being former members of the Sgr dSph galaxy. While our result for Pal 12 confirms a previous (independent) detection by Martínez-Delgado et al. (2002), this is the first time that Sgr Stream stars are found around NGC 4147. A thorough analysis of the structure and the stellar populations of the Sgr Stream will be presented when the full-sky 2MASS catalogs become available.

Note added in proof: While this paper was in the final stage of the peer review process, the 2MASS All Sky Catalogue has been released and a preprint was posted (Majewski et al. 2003, ApJ, submitted [astro-ph/0304198]) in which the Sgr Stream is traced all over the sky using the same tracer (M giants) used in the present paper. The results presented in this paper are fully confirmed by the complete 2MASS All Sky Catalogue.

Acknowledgements. M.B. and F.R.F. acknowledge the financial support to this research by the italian Ministero dell'Universitá e della Ricerca Scientifica (MURST) through the grant p. 2001028879, assigned to the project Origin and Evolution of Stellar Populations in the Galactic Spheroid. We are grateful to V. Marsakov for calling our attention to the new distance modulus of Ter 3, and to the Referee (M. Mateo) for several useful comments. This research has made use of NASA's Astrophysics Data System Abstract Service. M.B. dedicates this work to the memory of his father Giocondo.

\section{References}

Alard, C. 2001, A\&A, 377, 389

Bellazzini, M., Ferraro, F. R., \& Ibata, R. 2003, AJ, 125, 188 [BFI03]

Cole, A. A. 2001, ApJ, 559, L17

Dohm-Palmer, R. C., Helmi, A., Morrison, H., et al. 2001, ApJ, 555, L37

Dinescu, D. I., Majewski, S. R., Girard, T. M., \& Cudworth, K. M. 2000, AJ, 120, 1892
Fahlman, G. G., Mandushev, G., Richer, H. B., Thompson, I. B., \& Sivaranakrishnan, A. 1996, ApJ, 459, L65

Harris, W. E. 1996, AJ, 112, 1487

Helmi, A., White, S., de Zeeuw, P. T., \& Zhao, H. 1999, Nature, 402, 53

Ibata, R. A., Irwin, M. J., \& Gilmore, G. 1994, Nature, 370, 194

Ibata, R. A., Wyse, R. F. G., Gilmore, G., Irwin, M. J., \& Suntzeff, N. B. 1997, AJ, 113, 634

Ibata, R. A., \& Lewis, G. F. 1998, ApJ, 500, 575 (IL98)

Ibata, R. A., Lewis, G. F., Irwin, M., Totten, E., \& Quinn, T. 2001a, ApJ, 551, 294

Ibata, R. A., Irwin, M., Lewis, G. F., \& Stolte, A. 2001b, ApJ, 547, L136

Ibata, R. A., Lewis, G. F., Irwin, M., \& Cambrésy, L. 2002, MNRAS, 332,921

Ivezic, Z., Goldston, J., Finlator, K., et al. 2000, AJ, 120, 963

Johnston, K. V., Zhao, H., Spergel, D. N., \& Hernquist, L. 1999, ApJ, 512, L109

Kleyna, J. T., Geller, M. J., Kenyon, S. J., Kurtz, M. J., \& Thorstensen, J. R. 1998, AJ, 115, 2368

Kundu, A., et al. 2002, ApJ, L125

Majewski, S. R., Siegel, M. H., Kunkel, W. E., et al. 1999, AJ, 118, 1709

Martínez-Delgado, D., Aparicio, A., Gómez-Flechoso, M. A., \& Carrera, R. 2001, ApJ, 549, L199

Martínez-Delgado, D., Zinn, R., Carrera, R., \& Gallart, C. 2002, ApJ, 537, L19

Mateo, M. 1998, ARA\&A, 36, 435

Mateo, M., Nemec, J., Irwin, M., \& McMahon, R., AJ, 101, 892

Mateo, M., Olszewski, E. W., \& Morrison, H. L. 1998, ApJ, 508, L55

Monaco, L., Ferraro, F. R., Bellazzini, M., \& Pancino, E. 2002, ApJ, 578, L47

Newberg, H. J., Yanny, B., Rockosi, C., et al. 2002, ApJ, 569, 245

Renzini, A. 1998, AJ, 115, 2465

Renzini, A., \& Buzzoni, A. 1986, in Spectral Evolution of Galaxies, ed. C. Chiosi, \& A. Renzini (Drodrecht: Reidel), 135

Renzini, A., \& Fusi Pecci, F. 1988, ARA\&A, 26, 199

Savage, B. D., \& Mathis, J. S. 1979, ARA\&A, 17,73

Schlegel, D., Finkbeiner, D., \& Davis, M. 1998, ApJ, 500, 525

Vivas, A. K., et al. 2001, ApJ, 554, L33

Yanny, B., Newberg, H. J., Kent, S., et al. 2000, ApJ, 540, 825

Yanny, B., Newberg, H. J., Grebel, E. K., et al. 2003, ApJ, in press [astro-ph/0301029] 